10

\title{
Об экспериментальном определении параметров интенсивности $4 f-4 f$-переходов по спектрам излучения соединений европия (III)
}

\author{
(C) Lucca Blois ${ }^{1}$, Albano N. Carneiro Neto ${ }^{2}$, Ricardo L. Longo ${ }^{3}$, Israel F. Costa ${ }^{1}$, \\ Tiago B. Paolini ${ }^{1}$, Hermi F. Brito ${ }^{\top 1}$, Oscar L. Malta $\llbracket \uparrow 3$ \\ ${ }^{1}$ Department of Fundamental Chemistry, Institute of Chemistry, University of São Paulo, \\ São Paulo, Brazil \\ ${ }^{2}$ Physics Department and CICECO - Aveiro Institute of Materials, University of Aveiro, \\ Aveiro, Portugal \\ ${ }^{3}$ Department of Fundamental Chemistry, Federal University of Pernambuco, \\ Recife, Brazil \\ e-mail: ` hefbrito@iq.usp.br, ฯฯ oscar.malta@ufpe.br
}

Поступила в редакцию 16.07.2021 г.

В окончательной редакции 16.07.2021 г.

Принята к публикации 16.08.2021 г.

Комплексы Еu ${ }^{3+}$ и особенно $\beta$-дикетонаты хорошо известны благодаря их люминесцентным свойствам; они широко используются, в частности, в сенсорах и устройствах для освещения. Уникальной особенностью иона $\mathrm{Eu}^{3+}$ является возможность экспериментального определения параметров интенсивности $\Omega_{\lambda} 4 f-4 f$-переходов непосредственно из спектра излучения. Уравнения для определения $\Omega_{\lambda}$ по спектрам люминесценции отличаются при использовании детекторов излучаемой мощности и современных детекторов счета фотонов. Показано, что неправильное применение уравнений приводит к различию между величинами $\Omega_{\lambda}$, значительному для $\Omega_{4}$ (около $15.5 \%$ ) для нескольких комплексов $\beta$-дикетоната $\mathrm{Eu}^{3+}$, и к отличиям внутреннего квантового выхода $Q_{\mathrm{Ln}}^{\mathrm{Ln}}$, составляющим около $5 \%$. Из-за уникальных особенностей трехвалентных ионов лантанидов, таких как экранирование $4 f$-электронов, которое приводит к слабому влиянию ковалентности и кристаллического поля, наблюдается линейная корреляция между $\Omega_{\lambda}$, полученными с использованием уравнений для излучаемой мощности и счета фотонов. Подчеркнуто, что следует проявлять осторожность при выборе типа регистрации и предусматривать поправочные коэффициенты для параметров интенсивности. Предложено при вычислении $\Omega_{\lambda}$ определять интегральную интенсивность (пропорциональную площади полосы излучения) и центроид (или центр тяжести) перехода по правильно преобразованному по Якоби спектру в волновых числах (или энергии). Из-за небольшой ширины полос излучения типичных $4 f-4 f$-переходов площади и центроиды полос не зависят от преобразования в пределах экспериментальной погрешности. Эти оценки верны, поскольку они подтверждают ранее определенные $\Omega_{\lambda}$ без надлежащего преобразования спектра.

Ключевые слова: параметры интенсивности, теория Джадда-Офельта, поправочный коэффициент, излучаемая мощность, счет фотонов.

DOI: $10.21883 /$ OS.2022.01.51909.35-21

\section{1. Введение}

Соединения, содержащие лантаниды (или лантаноиды), окружают нас в повседневной жизни во многих технологиях, от МРТ-аппаратов до светодиодов, используемых для освещения или дисплеев [1-3]. Широкое применение таких соединений обусловлено уникальными свойствами, особенно фотофизическими и оптическими, ионов трехвалентных лантанидов $\left(\mathrm{Ln}^{3+}\right)$, в исследование которых профессор Марина Попова внесла существенный вклад [4-8]. Одной из особенностей ионов $\mathrm{Ln}^{3+}$ является возможность полного описания интенсивностей $f-f$-переходов только тремя величинами, известными как параметры интенсивности [9-11]. Эти параметры важны для количественной оценки таких фотофизических свойств, как коэффициенты ветвления и излучательное время жизни, что применяется в лазерах и люминесцентных материалах. Поэтому грамотное экс- периментальное определение параметров интенсивности важно для дальнейших разработок и усовершенствований материалов на основе лантанидов, а также для проверки теоретических моделей [11-14]. Среди элементов ряда лантанидов европий играет важную роль в фотонике и оптических применениях как ион $\mathrm{Eu}^{3+}$, так и $\mathrm{Eu}^{2+}$, каждый из которых имеет собственные спектроскопические характеристики. Благодаря особенностям электронной структуры иона $\mathrm{Eu}^{3+}$ спектры излучения соединений и материалов, содержащих этот ион, могут быть использованы для определения параметров интенсивности $[10,12,15]$. В то же время для иона $\mathrm{Eu}^{2+}$ характерны разрешенные дипольные переходы $4 f-5 d$; он был применен, например, в материалах, которые демонстрируют явление длительной люминесценции с возможностью перестраивания цвета излучения за счет эффектов сильного поля лиганда [16-18]. Однако элек- 
тронные переходы $\mathrm{Eu}^{3+}$, а также большинства ионов $\mathrm{Ln}^{3+}$ имеют $4 f-4 f$-природу и, следовательно, являются запрещенными как электродипольные, с намного более низкими силами осцилляторов и более продолжительным временем жизни возбужденного состояния, что позволяет широкое использование ионов $\mathrm{Ln}^{3+}$ : от нанотермометров до маркеров безопасности [19-22]. Проблема низких сил осцилляторов $4 f-4 f$-переходов ионов $\mathrm{Ln}^{3+}$ может быть решена, как показал Вайсман в 1942 г. [23], путем координации $\mathrm{Ln}^{3+}$ с хромофорами, которые могут поглощать свет и способствовать эффективному заселению возбужденных уровней иона, что обусловливает получение люминесцентных материалов с высокой интенсивностью излучения.

Ключом к пониманию $4 f-4 f$-интенсивностей трехвалентных ионов лантанида являются вынужденный электрический дипольный механизм (FED) и механизм динамической связи (DC). В 1962 г. Джадд и Офельт независимо представили механизм FED, который удовлетворительно объяснил природу переходов $4 f-4 f$ [24,25]. Однако механизм FED содержал несоответствия, касающиеся так называемых сверхчувствительных переходов, свидетельствуя о том, что может существовать еще один важный механизм. Так, в 1964 г. Йоргенсен и Джадд представили псевдоквадрупольный механизм для объяснения поведения этих сверхчувствительных переходов [26]. В 1975 г. Мейсон с соавторами ввели формализм и термин „механизм динамической связи“ [27], который относится к эффекту, зависящему от поляризуемости лиганда. Однако Джадд продемонстрировал в 1979 г., что DC-механизм, предложенный Мейсоном, формально идентичен его предыдущей работе с Йоргенсеном [28].

Для нецентросимметричных систем влияние нечетных компонент кристаллического поля проявляется как возмущение к гамильтониану свободного иона. В результате возмущенные волновые функции $4 f$-состояний имеют компоненты с противоположной четностью, соответствующие $5 d, 6 d, 5 g$ и т.д. [24,25]. Это приводит к выражениям для скоростей переходов, которые преобразуют эту смесь конфигураций, вызванную полем лиганда, в так называемые параметры интенсивности Джадда-Офельта (или параметры интенсивности), часто обозначаемые как $\Omega_{\lambda}(\lambda=2,4$, и 6$)$, которые могут быть измерены экспериментально. В соответствии с DC-механизмом возбуждающее излучение индуцирует осциллирующие электрические поля на атомах лиганда, пропорциональные поляризуемости. Таким образом, индуцированное поле может активизировать $4 f-4 f$-переходы, и окончательное уравнение для скоростей переходов будет таким, как в теории Джадда-Офельта, т. е. нельзя экспериментально определить вклад каждого механизма в параметры интенсивности $\Omega_{\lambda}[11]$. Экспериментальное определение параметров интенсивности имеет большое значение при изучении локального окружения иона лантанида; в сочетании с моделями поля лигандов можно получить информацию о структуре и связи между ионом и первой координационной сферой, такую как заряды, поляризуемость и ковалентность $[14,29]$. Сообщалось даже о дальнодействующей структурной чувствительности для параметров $\Omega_{\lambda}$ в некоторых соединениях [12].

В случае иона $\mathrm{Eu}^{3+}$ экспериментальное определение параметров интенсивности упрощается благодаря структуре энергетических уровней этого иона. Так как излучающий уровень ${ }^{5} D_{0}$ имеет нулевой полный угловой момент, то в соответствии с правилами отбора на интенсивность ${ }^{5} D_{0} \rightarrow{ }^{7} F_{2}$-перехода влияет только $\Omega_{2}$, на интенсивность ${ }^{5} D_{0} \rightarrow{ }^{7} F_{4}-\Omega_{4}$, а на интенсивность ${ }^{5} D_{0} \rightarrow{ }^{7} F_{6}-\Omega_{6}$. Кроме того, ионы $\mathrm{Eu}^{3+}$ имеют переход ${ }^{5} D_{0} \rightarrow{ }^{7} F_{1}$, который является практически магнитодипольным, что делает этот переход почти независимым от поля лигандов и зависящим от показателя преломления. Этот переход можно использовать в качестве внутреннего эталона для расчета скоростей спонтанного излучения переходов ${ }^{5} D_{0} \rightarrow{ }^{7} F_{J}(J=2,4$ и 6).

Одними из наиболее эффективно люминесцирующих соединений $\mathrm{Eu}^{3+}$ являются комплексы на основе $\beta$-дикетонатов [10]. Этот класс соединений широко изучен, поскольку они, как правило, обладают высокой интенсивностью излучения при возбуждении в полосу поглощения лигандов благодаря эффективной сенсибилизации ионов $\mathrm{Eu}^{3+}$. Это может обеспечить квантовый выход люминесценции примерно до $80 \%$, хотя большинство комплексов не выдерживают ультрафиолетовое облучение в течение длительного времени [9,20,21,23,30-33]. Даже при синтезе новых лигандов во многих случаях основной частью люминесцирующего комплекса является фрагмент $\beta$-дикетонового типа [34].

При расчёте большинства параметров по спектрам излучения соединений $\mathrm{Eu}^{3+}$ в в последние два десятилетия использовалась регистрация излучаемой мощности, в то время как большинство современного оборудования позволяет определять количество излучаемых фотонов в секунду [35-42]. Кроме того, все оценки параметров интенсивности, интегральных интенсивностей и центроидов получали при использовании спектров излучения, записанных в длинах волн. Однако для их правильного определения следует использовать спектры излучения в шкале волновых чисел (или энергии), что требует преобразования сигнала $[43,44]$. В настоящей работе получено несколько комплексов $\beta$-дикетонатов $\mathrm{Eu}^{3+}$, для которых зарегистрированы спектры фотолюминесценции. По спектрам излучения определены экспериментальные параметры интенсивности $\Omega_{\lambda} 4 f-4 f$-переходов с использованием выражений для детекторов счета фотонов и сопоставлены с данными, полученными с использованием уравнения для излучаемой мощности, a также с учетом соответствующих преобразований спектров в волновые числа. 


\section{2. Экспериментальная часть}

2-Теноилтрифторацетон (Htta, 99\%), бензоилтрифторацетон (Hbtfa, 99\%), 1,10-фенантролин (phen, 99\%), хлорид тетраэтиламмония $\left(\mathrm{Et}_{4} \mathrm{NCl},>98 \%\right)$ и хлорид 1-этил-3-метилимидазолия $\left(\mathrm{C}_{2} \mathrm{mimCl},>98 \%\right)$ приобретены у Merck и использованы без дальнейшей очистки. $\mathrm{Eu}_{2} \mathrm{O}_{3}$ приобретен у CSTARM $(99.99 \%)$ и превращен в $\mathrm{EuCl}_{3} \cdot 6 \mathrm{H}_{2} \mathrm{O}$ в соответствии с процедурой, ранее описанной в литературе [36].

Микроанализ проводился на Perkin-Elmer 2400 серии II, ESI-MS-спектры регистрировали на спектрометре Amazon speed ETD Bruker Daltonics с детектором Ion Trap, с использованием источника ионизации электрораспылением $4500 \mathrm{~V}$ под потоком горячего газа $\mathrm{N}_{2}$ $\left(300^{\circ} \mathrm{C}, 5 \mathrm{~L} / \mathrm{min}\right)$. Спектры люминесценции регистрировали на приборе Edinburgh FLS980, используя в качестве источника возбуждения дуговую Хе-лампу мощностью $450 \mathrm{~W}$.

\section{1. Синтез комплексов $\left[\mathrm{Eu}(\beta \text {-dik })_{3}(\mathrm{~L})_{n}\right]$}

Все комплексы синтезированы по единой методике, адаптированной из литературы $[9,15]$. Первоначально раствор $3 \mathrm{mmol} \mathrm{NaOH}$ в $10 \mathrm{~mL}$ деионизированной воды по каплям добавляли к этанольному раствору $3 \mathrm{mmol}$ $\beta$-дикетона (Htta или Hbtfa) при постоянном перемешивании при $60^{\circ} \mathrm{C}$. Затем к этой смеси по каплям добавляли водный раствор $\mathrm{EuCl}_{3} \cdot 6 \mathrm{H}_{2} \mathrm{O}(1 \mathrm{mmol}$ в $10 \mathrm{~mL}$ деионизированной воды). После частичного испарения этанола выделялся комплекс $\left[\mathrm{Eu}(\beta \text {-dik })_{3}\left(\mathrm{H}_{2} \mathrm{O}\right)_{n}\right]$ в виде масла при образовании двух фаз. Затем при интенсивном перемешивании добавляли $20 \mathrm{~mL}$ деионизированной воды для осаждения твердой субстанции $\left[\mathrm{Eu}(\beta \text {-dik })_{3}\left(\mathrm{H}_{2} \mathrm{O}\right)_{n}\right]$. После фильтрования комплекс сушили при $50^{\circ} \mathrm{C}$ в течение $72 \mathrm{~h}$.

Молекулы воды заменяли на 1,10-фенантролин следующим образом: $0.5 \mathrm{mmol}\left[\mathrm{Eu}(\beta \text {-dik })_{3}\left(\mathrm{H}_{2} \mathrm{O}\right)_{n}\right]$ растворяли в $20 \mathrm{~mL}$ этанола с последующим добавлением по каплям этанольного раствора 1,10-фенантролина $(1 \mathrm{mmol}$ в $10 \mathrm{~mL}$ этанола) при постоянном перемешивании. Комплекс $\left[\mathrm{Eu}(\beta \text {-dik })_{3}(\mathrm{phen})\right]$ выделялся почти мгновенно. Смесь перемешивали в течение $2 \mathrm{~h}$, а затем помещали в ледяную баню для осаждения комплекса. Образовавшиеся соединения отфильтровывали и сушили при $50^{\circ} \mathrm{C}$ в течение $72 \mathrm{~h}$.

$\left[\mathrm{Eu}(\mathrm{tta})_{3}\left(\mathrm{H}_{2} \mathrm{O}\right)_{2}\right] \mathrm{ESI}(+) \mathrm{MS}: m / z[\mathrm{M}+3 \mathrm{H}]^{+}=852.91$, ca. 852.91. Аналитический расчет для $\mathrm{C}_{24} \mathrm{H}_{16} \mathrm{EuF}_{9} \mathrm{O}_{8} \mathrm{~S}_{3}$ : С 33.85, Н 1.89\%, найдено: С 34.06, Н $1.85 \%$.

$\left[\mathrm{Eu}(\mathrm{btfa})_{3}\left(\mathrm{H}_{2} \mathrm{O}\right)_{2}\right] \mathrm{ESI}(+) \mathrm{MS:} m / z[\mathrm{M}+3 \mathrm{H}]^{+}=835.01$, са. 835.05. Аналитический расчет для $\mathrm{C}_{30} \mathrm{H}_{20} \mathrm{EuF}_{9} \mathrm{O}_{7}$ : С 43.23, Н 2.66\%, найдено: С 43.10, Н 2.68\%.

$$
\left[\mathrm{Eu}(\mathrm{tta})_{3}(\text { phen })\right] \quad \operatorname{ESI}(+) \quad \text { MS: }
$$

$m / z \quad\left[\mathrm{Eu}(\mathrm{tta})_{2}(\text { phen })_{2}\right]^{+}=953.11, \quad$ ca. $\quad 953.03$.

Аналитический расчет для $\mathrm{C}_{36} \mathrm{H}_{20} \mathrm{EuF}_{9} \mathrm{~N}_{2} \mathrm{O}_{6} \mathrm{~S}_{3}$ : C 43.43 , Н 2.02, N 2.81\%, найдено: С 43.23, Н 1.99, N 2.82\%.

$$
\left[\mathrm{Eu}(\mathrm{btfa})_{3}(\text { phen })\right] \quad \operatorname{ESI}(+) \quad \text { MS: }
$$

$\mathrm{m} / z \quad\left[\mathrm{Eu}(\mathrm{btfa})_{2}(\text { phen })_{2}\right]^{+}=941.20, \quad$ ca. $\quad 941.12$. Аналитический расчет для $\mathrm{C}_{42} \mathrm{H}_{26} \mathrm{EuF}_{9} \mathrm{~N}_{2} \mathrm{O}_{6}$ : C 51.60 , Н 2.68, N 2.87\%, найдено: С 51.27, Н 2.73, N 2.99\%.

\section{2. Синтез комплексов $Q\left[\operatorname{Eu}(\beta \text {-dik })_{4}\right]$}

Все комплексы синтезированы в соответствии с методикой, о которой ранее сообщала наша группа [21]. К изопропанольному раствору 2-теноилтрифторацетона $\mathrm{Htta}(20 \mathrm{mmol}$ в $40 \mathrm{~mL}$ iPrOH$)$ при перемешивании при $60^{\circ} \mathrm{C}$ по каплям добавляли водный раствор гидроксида натрия $\mathrm{NaOH}\left(20 \mathrm{mmol}\right.$ в $\left.10 \mathrm{~mL} \mathrm{H} \mathrm{H}_{2} \mathrm{O}\right)$. Затем добавляли $10 \mathrm{~mL}$ изопропанольного раствора хлорида тетраэтиламмония $\mathrm{Et}_{4} \mathrm{~N}^{+} \mathrm{Cl}^{-}(6 \mathrm{mmol})$. В конце добавляли водный раствор хлорида европия (III) (4 mmol в $10 \mathrm{~mL}$ воды), после чего образовывался осадок $Q\left[\operatorname{Eu}(\beta \text {-dik })_{4}\right]$. После перемешивания при температуре около $70^{\circ} \mathrm{C}$ в течение $2 \mathrm{~h}$ смесь фильтровали, промывали холодным этанолом и сушили при пониженном давлении в течение $5 \mathrm{~h}$. Анализы ESI-MS проводили в отрицательном режиме для поиска аниона $\left[\mathrm{Eu}(\beta \text {-dik })_{4}\right]^{-}$.

$$
\mathrm{Et}_{4} \mathrm{~N}^{+}\left[\mathrm{Eu}(\mathrm{tta})_{4}\right] \quad \mathrm{ESI}(-) \quad \mathrm{MS} \text { : }
$$
$m / z\left[{ }^{153} \mathrm{Eu}(\mathrm{tta})_{4}\right]^{-}=1036.87$, са 1036.96 Аналитический расчет для $\mathrm{C}_{40} \mathrm{H}_{36} \mathrm{EuF}_{12} \mathrm{NO}_{8} \mathrm{~S}_{4}$ : C 41.17, $\mathrm{H} 3.11, \mathrm{~N} 1.20 \%$, найдено: С 41.10, Н 3.10, N 1.24\%.

$$
\mathrm{C}_{2} \operatorname{mim}\left[\mathrm{Eu}(\mathrm{tta})_{4}\right] \quad \operatorname{ESI}(-)
$$

MS: $m / z\left[{ }^{153} \mathrm{Eu}(\mathrm{tta})_{4}\right]^{-}=1036.94$, са. 1036.96 Аналитический расчет для $\mathrm{C}_{38} \mathrm{H}_{27} \mathrm{EuF}_{12} \mathrm{~N}_{2} \mathrm{O}_{8} \mathrm{~S}_{4}:$ C $39.76, \mathrm{H}$ 2.37, N 2.44\%, найдено: С 39.77, Н 2.34, N 2.44\%.

\section{3. Результаты и обсуждение}

Результаты элементного анализа образцов хорошо согласуются с предлагаемыми формулами соединений. Результаты ESI-MS (рис. S1-S10) также указывают на то, что мы получили желаемые комплексы, хотя стоит отметить, что комплексы лантанидов могут вступать в реакцию во время ионизации даже в мягких условиях, таких как ионизация электрораспылением, как это, по-видимому, произошло с соединениями $\left[\mathrm{Eu}(\beta \text {-dik) })_{3}(\right.$ phen $\left.)\right] \quad[45]$. Предлагается реакция обмена лигандами, такая как $2\left[\operatorname{Eu}(\beta \text {-dik })_{3}(\right.$ phen $\left.)\right] \rightarrow\left[\operatorname{Eu}(\beta \text {-dik })_{2}(\text { phen })_{2}\right]^{+}+$ $+\left[\operatorname{Eu}(\beta \text {-dik })_{4}\right]^{-}$, из-за присутствия $\left[\operatorname{Eu}(\beta \text {-dik })_{4}\right]^{-}$ионов в спектре ESI (-) фенантролиновых комплексов (рис. S3 и S10), хотя необходимы дальнейшие исследования.

Спектры люминесценции всех комплексов представлены на рис. 1. Спектры зарегистрированы для соединений в твердом состоянии (порошкообразные образцы) при комнатной температуре $(298 \mathrm{~K})$ в диапазоне от 570 до $720 \mathrm{~nm}$ при возбуждении в наиболее интенсивную полосу лиганда. Все соединения показали наличие сигнала в области сверхчувствительного перехода ${ }^{5} D_{0} \rightarrow{ }^{7} F_{2}$, а также других переходов $\mathrm{Eu}^{3+}$ 

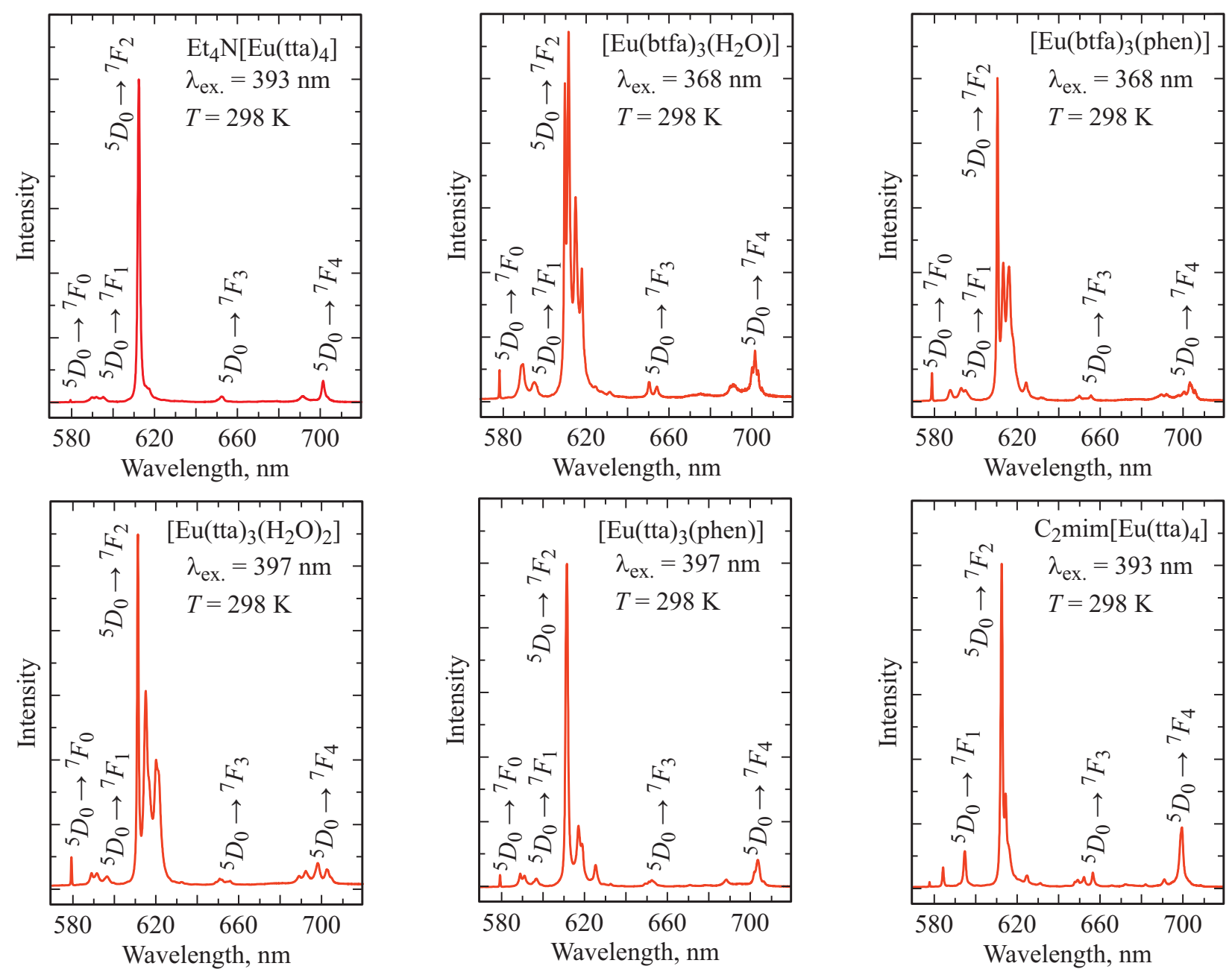

Рис. 1. Спектры люминесценции комплексов $\left[\operatorname{Eu}(\beta \text {-dik })_{3}(\mathrm{~L})_{n}\right]$ и $\mathrm{Q}\left[\operatorname{Eu}(\beta \text {-dik })_{4}\right]$ при возбуждении в максимуме полосы лиганда при $298 \mathrm{~K}$.

${ }^{5} D_{0} \rightarrow{ }^{7} F_{0,1,3,4}$ в виде узких полос излучения. Интересной особенностью тетракис-комплексов является отсутствие перехода ${ }^{5} D_{0} \rightarrow{ }^{7} F_{0}$, что указывает на точечную группу симметрии, отличную от $\mathrm{C}_{n(v)}$. Также следует отметить, что $\mathrm{Et}_{4} \mathrm{~N}\left[\mathrm{Eu}(\mathrm{tta})_{4}\right]$ имеет почти монохроматический переход ${ }^{5} D_{0} \rightarrow{ }^{7} F_{2}$ с полушириной линии $1.250 \mathrm{~nm}\left(33 \mathrm{~cm}^{-1}\right)$. Спектры возбуждения соединений представлены на рис. S11.

По спектрам излучения комплексов можно рассчитать параметры интенсивности $\Omega_{\lambda}$ переходов $4 f-4 f$ иона $\mathrm{Eu}^{3+}$ (таблица). Чтобы выявить разницу между параметрами, полученными с использованием формулы для излучаемой мощности в случае детектора счета фотонов, полезно сначала представить известные уравнения для определения таких параметров, начиная с определения спектральной интенсивности перехода в терминах излучаемой мощности, $I^{\mathrm{P}}$, и счета фотонов, $I^{\mathrm{C}}$ :

$$
\begin{gathered}
I_{0 J}^{\mathrm{P}}=N A_{0 J} \hbar \omega_{0 J}=2 \pi N A_{0 J} \hbar c \sigma_{0 J} \\
I_{0 J}^{\mathrm{C}}=N A_{0 J}
\end{gathered}
$$

Экспериментальные параметры интенсивности $4 f-4 f$-переходов (в $\left.10^{-20} \mathrm{~cm}^{2}\right)$, полученные с использованием выражений для мощности излучения, $\Omega_{\lambda}^{\mathrm{P}}$, и счета фотонов, $\Omega_{\lambda}^{\mathrm{C}}$, и соответствующий внутренний квантовый выход, $Q_{\mathrm{Ln}}^{\mathrm{Ln}}($ в \%)

\begin{tabular}{c|c|c|c|c|c|c}
\hline Комплекс & $\Omega_{2}^{\mathrm{P}}$ & $\Omega_{2}^{\mathrm{C}}$ & $\Omega_{4}^{\mathrm{P}}$ & $\Omega_{4}^{\mathrm{C}}$ & $Q_{\mathrm{Ln}}^{\mathrm{Ln}}(\mathrm{P})$ & $Q_{\mathrm{Ln}}^{\mathrm{Ln}}(\mathrm{C})$ \\
\hline$\left[\mathrm{Eu}(\mathrm{tta})_{3}\left(\mathrm{H}_{2} \mathrm{O}\right)_{2}\right]$ & 33 & 32 & 8.0 & 6.8 & 32 & 30 \\
\hline$\left.[\mathrm{Eu}(\mathrm{tta})]_{3}(\mathrm{phen})\right]$ & 22 & 21 & 5.0 & 4.2 & 63 & 60 \\
\hline$\left[\mathrm{Eu}(\mathrm{btfa})_{3}\left(\mathrm{H}_{2} \mathrm{O}\right)_{2}\right]$ & 20 & 19 & 7.1 & 6.0 & 33 & 32 \\
\hline$\left[\mathrm{Eu}(\mathrm{btfa})_{3}(\mathrm{phen})\right]$ & 24 & 23 & 5.8 & 4.9 & 63 & 60 \\
\hline $\mathrm{Et}_{4} \mathrm{~N}\left[\mathrm{Eu}(\mathrm{tta})_{4}\right]$ & 30 & 28 & 9.5 & 8.0 & 98 & 93 \\
\hline $\mathrm{C}_{2} \mathrm{mim}\left[\mathrm{Eu}(\mathrm{tta})_{4}\right]$ & 15 & 14 & 10 & 8.6 & 52 & 49
\end{tabular}

где $N$ - заселенность излучательного уровня, $A_{0 J}-$ коэффициент спонтанного излучения перехода ${ }^{5} D_{0} \rightarrow{ }^{7} F_{J}$, $\omega_{0 J}-$ угловая частота, $\sigma_{0 J}-$ центроид перехода в волновых числах $\left(\right.$ в $\left.\mathrm{cm}^{-1}\right), \hbar-$ приведенная постоянная Планка, $c$ - скорость света.

Из теорий Джадда-Офельта и динамической связи можно получить выражения для коэффициентов спон- 
танного излучения для электродипольных переходов иона $\mathrm{Eu}^{3+}$ в виде

$$
A_{0 \lambda}=\Lambda \sigma_{0 \lambda}^{3} \Omega_{\lambda}, \quad \Lambda=\frac{32 \pi^{3} e^{2} \chi}{3 \hbar}\left|\left\langle{ }^{5} D_{0} \| U^{(\lambda)} \mid{ }^{7} F_{\lambda}\right\rangle\right|^{2},
$$

где $e-$ элементарный заряд, $\chi-$ поправочный коэффициент локального поля Лоренца, $\Omega_{\lambda}$ - параметр интенсивности $4 f-4 f$-перехода и $\left\langle{ }^{5} D_{0}|| U^{(\lambda)} \|{ }^{7} F_{\lambda}\right\rangle^{2}-$ приведенный матричный элемент унитарного тензорного оператора ранга $\lambda$ со значениями 0.0032 для $\lambda=2$ и 0.0023 для $\lambda=4$ для иона $\mathrm{Eu}^{3+}$ [46]. Следует отметить, что неверно напечатанное значение 0.032 матричного элемента для $\lambda=4$ было обнаружено в широко используемых и цитируемых работах $[19,47]$.

Для разрешенного магнитодипольного и запрещенного электродипольного перехода ${ }^{5} D_{0} \rightarrow{ }^{7} F_{1}(\Delta J=1)$ скорость спонтанного излучения может быть выражена как

$$
A_{01}=\kappa \sigma_{01}^{3},
$$

где $\kappa=0.31 \cdot 10^{-11} n^{3}$ с показателем преломления среды $n$ (Supporting information). В настоящей работе $n$ считали равным 1.5 для всех соединений.

При известном значении $A_{01}$ можно рассчитать все остальные $A_{0 \lambda}$ по соотношению интенсивностей излучения, которые пропорциональны проинтегрированным областям в спектре излучения, а именно

$$
\begin{gathered}
\frac{I_{0 \lambda}^{\mathrm{P}}}{I_{01}^{\mathrm{P}}}=\left(\frac{S_{0 \lambda}}{S_{01}}\right)=\left(\frac{A_{0 \lambda}}{A_{01}}\right)\left(\frac{\sigma_{0 \lambda}}{\sigma_{01}}\right)=\frac{\Lambda}{\kappa} \frac{\sigma_{0 \lambda}^{4} \Omega_{\lambda}^{\mathrm{P}}}{\sigma_{01}^{4}}, \\
\frac{I_{0 \lambda}^{\mathrm{C}}}{I_{01}^{\mathrm{C}}}=\left(\frac{S_{0 \lambda}}{S_{01}}\right)=\left(\frac{A_{0 \lambda}}{A_{01}}\right)=\frac{\Lambda}{\kappa} \frac{\sigma_{0 \lambda}^{3} \Omega_{\lambda}^{\mathrm{C}}}{\sigma_{01}^{3}},
\end{gathered}
$$

где $S_{0 \lambda}$ - площадь полосы излучения, соответствующая переходам ${ }^{5} D_{0} \rightarrow{ }^{7} F_{\lambda}(\lambda=2,4$ или 6), верхние индексы Р и С обозначают, что спектр зарегистрирован, как счет фотонов, но вычисления производились по математической формуле для излучаемой мощности и счета фотонов соответственно.

Внутренний квантовый выход излучения $Q_{\mathrm{Ln}}^{\mathrm{Ln}}-$ это величина, которая определяет внутреннюю эффективность иона $\mathrm{Ln}^{3+}$ как излучающего центра. Таким образом, $Q_{\mathrm{Ln}}^{\mathrm{Ln}}$ можно рассчитать следующим образом:

$$
Q_{\mathrm{Ln}}^{\mathrm{Ln}}=\frac{A_{\mathrm{rad}}}{A_{\mathrm{rad}}+A_{\text {rad }}}=A_{\mathrm{rad}} \tau,
$$

где $A_{\mathrm{rad}}$ - сумма по всем излучательным скоростям, a $A_{n \text { rad }}$ - скорость безызлучательного распада. Сумма этих скоростей соответствует обратной величине времени жизни $\left(A_{\mathrm{rad}}+A_{n \mathrm{rad}}=\tau^{-1}\right)$ в отсутствие процессов передачи энергии, связанных с излучательным уровнем. Полученные значения времени жизни ${ }^{5} D_{0}$ для исследуемых соединений представлены в табл. S1 и рис. S12. Для случая соединений, содержащих ион $\mathrm{Eu}^{3+}$, уравнение (7) может быть переписано как

$$
Q_{\mathrm{Ln}}^{\mathrm{Ln}}=\tau \sum_{\lambda} A_{0 \lambda}
$$

Таким образом, в зависимости от того, какой тип регистрации учитывался при расчетах $A_{0 \lambda}, Q_{\mathrm{Ln}}^{\mathrm{Ln}}$ может определяться с ошибками (в основном в коэффициентах излучения $A_{02}$ и $\left.A_{04}\right)$, если не применяется скорректированная формула. В таблице показаны, например, значения $Q_{\mathrm{Ln}}^{\mathrm{Ln}}$ с использованием формулы излучаемой мощности для спектров люминесценции, полученных с помощью оборудования для счета фотонов. Это может привести к завышению на 5\% правильных значений $Q_{\mathrm{Ln}}^{\operatorname{Ln}}(\mathrm{C})$ (рис. S13).

Важно подчеркнуть, что линейное поведение, наблюдаемое для корреляций на рис. 2, обусловлено эффектом экранирования заполненными подуровнями $5 s 5 p$ в ионах $\mathrm{Ln}^{3+}$. Так, $4 f$-электроны подвергаются чрезвычайно малому влиянию поля лиганда и исчезающе малому влиянию ковалентности, что делает центроиды переходов $4 f-4 f$ сильно локализованными. В результате соотношение между центроидами не претерпевает существенных изменений при сравнении различных соединений, что делает возможным существование линейного поправочного коэффициента.

Поправочный коэффициент получается путем сравнения математических выражений для зарегистрированной интенсивности в случаях излучаемой мощности и счета фотонов в виде соотношения между уравнениями (5) и (6), что дает следующее выражение для поправочного коэффициента:

$$
\Omega_{\lambda}^{\mathrm{C}}=\Omega_{\lambda}^{\mathrm{P}}\left(\frac{\sigma_{0 \lambda}}{\sigma_{01}}\right)
$$

где $\sigma_{0 \lambda}$ - центроид перехода ${ }^{5} D_{0} \rightarrow{ }^{7} F_{\lambda}$ с $\lambda=2,4$ или 6 .

Для анализируемых здесь комплексов $\beta$-дикетонатов $\mathrm{Eu}^{3+}$ мы получаем средний поправочный коэффициент с очень низкой погрешностью благодаря слабому влиянию ковалентности и кристаллического поля на $4 f$-электроны, а именно $\left\langle\frac{\sigma_{02}}{\sigma_{01}}\right\rangle=0.9636 \pm 0.0008$ и $\left\langle\frac{\sigma_{04}}{\sigma_{01}}\right\rangle=0.8478 \pm 0.0007$.

Стоит отметить, что сами параметры интенсивности $\Omega_{\lambda}$ не зависят от типа детектора. Так как эти параметры являются физически наблюдаемыми, они не могут зависеть от измерения. Поправочный коэффициент следует применять к параметрам интенсивности, полученным с помощью детекторов счета фотонов, но рассчитанным с использованием формулы для излучаемой мощности, которая использовалась в различных работах в последние 20 лет. То есть параметры, вычисленные с помощью соответствующих уравнений из спектра, записанного с регистрацией излучаемой мощности, должны быть такими же, как и параметры, рассчитанные по спектру, записанному с детектором счета фотонов, при условии, что для каждого случая используются правильные уравнения.

Как видно из всех уравнений, приведенных выше, площади и, что наиболее важно, центры тяжести (или центроиды), используемые для получения скоростей 

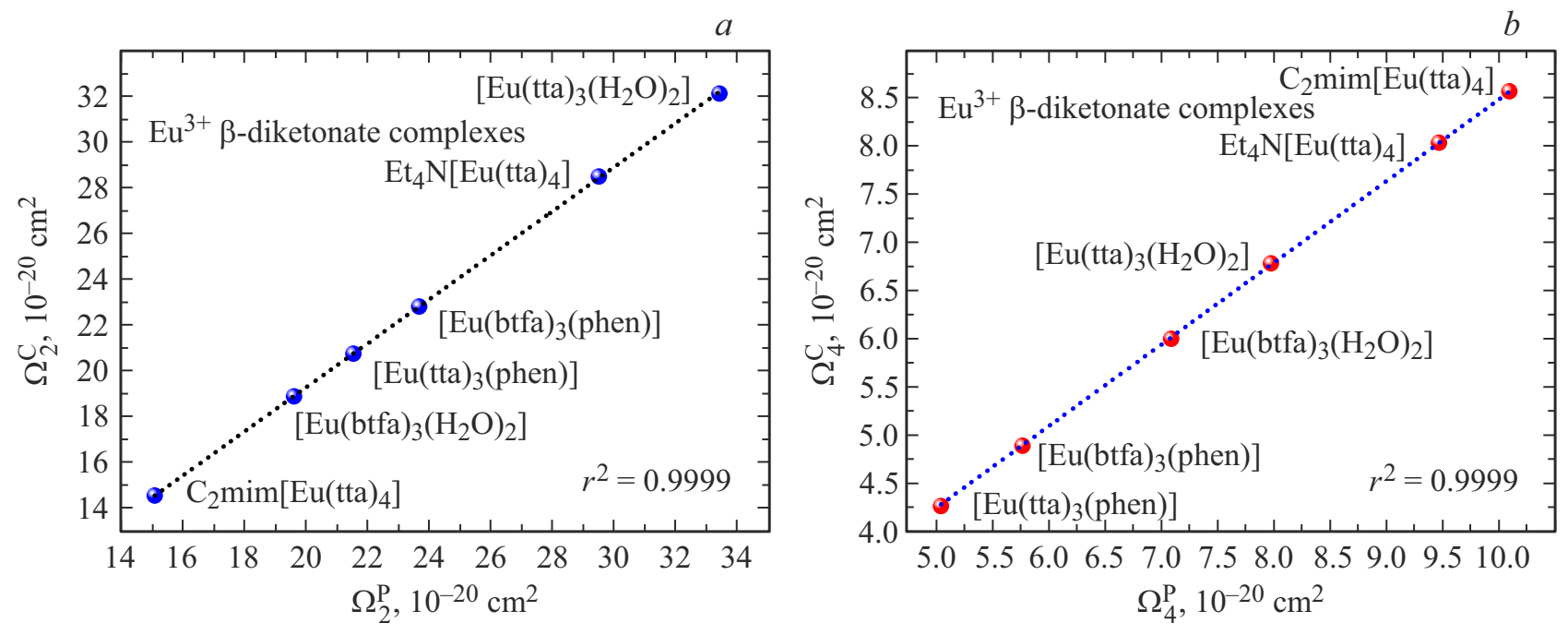

Рис. 2. Корреляция между параметрами интенсивности $4 f-4 f$-переходов комплексов $\beta$-дикетонатов Еu ${ }^{3+}$, рассчитанных с использованием выражений для излучаемой мощности $\left(\Omega^{\mathrm{P}}\right)$ и счета фотонов $\left(\Omega^{\mathrm{C}}\right)$.

излучения и параметров интенсивности, должны определяться по спектру в волновых числах (или энергии, или частоте). Однако поскольку спектры обычно записывают в длинах волн, их необходимо преобразовать, например, в волновые числа, а сигнал трансформировать с помощью так называемого преобразования Якоби $[43,44]$. Для широких полос излучения это преобразование оказывает значительное влияние на относительные интенсивности, положения максимумов и форму линий. Например, гауссова форма линии, наблюдаемая в спектре излучения $\mathrm{Ba}_{2} \mathrm{WO}_{3} \mathrm{~F}_{4}$ в волновых числах, становится заметно асимметричной в длинах волн [48]. Кроме того, $\lambda_{\max }=490 \mathrm{~nm}$ (максимум в спектре длин волн), что соответствует $20408 \mathrm{~cm}^{-1}$, тогда как $\tilde{v}_{\max }=19700 \mathrm{~cm}^{-1}$ (максимум в спектре волновых чисел), смотрите Appendix 4: Plotting Emission Spectra в работе [48], что дает значительную разницу (около $700 \mathrm{~cm}^{-1}$ ) между этими максимумами, определенными по правильному спектру и спектру в длинах волн. Насколько нам известно, это преобразование никогда не использовалось при определении параметров интенсивности по спектрам излучения, что побудило нас исследовать влияние этого преобразования.

Соотношение между энергией $E$ или частотой $v$, или угловой частотой $\omega$, или волновым числом $\tilde{v}$, представленное в общем виде через $\sigma$ и длину волны $\lambda$, может быть выражено как $\sigma=a \lambda^{-1}$, с $a=10^{7}$ для $\sigma \equiv \tilde{v}, \lambda$ в nm, $a=h c$ для $\sigma \equiv E$, или $a=c$ для $\sigma \equiv v$. Поэтому преобразование $\lambda$ в $\sigma$ является простым, однако следует отметить, что из-за нелинейной зависимости сбор равномерно распределенных данных в $\lambda$ приведет к сильно неравномерному распределению данных в $\sigma$, что может привести к различиям при выполнении численного интегрирования. По правилу цепочки $[43,44]$, можно написать: $f(\sigma)=f(\lambda) d \lambda / d \sigma$, где $f(\lambda)$ - сигнал, наблюдаемый при первоначальном поступлении данных в длинах волн, $f(\sigma)$ - преобразованный сигнал, когда длины волн трансформированы в $\sigma$. Объединяя эти два уравнения, можно получить $f(\sigma)=-a \sigma^{-2} f(\lambda)$, называемое преобразованием Якоби, где исходная функция $f(\lambda)$ должна быть умножена на коэффициент $-a \sigma^{-2}$, чтобы получить правильно преобразованный спектр. В частности, для преобразования в волновые числа: $\tilde{v}\left(\mathrm{~cm}^{-1}\right)=10^{7} / \lambda(\mathrm{nm})$ и $f(\tilde{v})=-10^{7} f(\lambda) / \tilde{v}^{2}$.

Мы вычислили площади интересующих нас переходов, используя исходный спектр, записанный со счетом фотонов, как функцию длины волны, а также преобразованный по Якоби спектр в волновых числах. Различия между этими площадями были меньше $1 \%$, что меньше экспериментальной погрешности в таких измерениях или даже погрешности в пределах интегрирования. Кроме того, на центроиды $\sigma_{0 \lambda}$ переходов ${ }^{5} D_{0} \rightarrow{ }^{7} F_{\lambda}$ преобразование спектра существенно не повлияло (табл. S2). В результате полученные значения параметров интенсивности были в пределах экспериментальной погрешности независимо от преобразования Якоби. Это важные оценки, поскольку они подтверждают все данные и значения параметров интенсивности, полученные ранее из спектров излучения, к которым не применялось преобразование Якоби. Эта нечувствительность к трансформации спектра в основном обусловлена малой шириной полос излучения, характерных для соединений на основе $\mathrm{Ln}^{3+}$.

\section{4. Выводы}

Синтезировано несколько комплексов $\beta$-дикетонатов $\mathrm{Eu}^{3+}$ с общими формулами $\left[\mathrm{Eu}(\beta \text {-dik })_{3}(\mathrm{~L})_{n}\right] \quad$ и $\mathrm{Q}\left[\mathrm{Eu}(\beta \text {-dik) })_{4}\right], \quad$ которые подтверждены элементным анализом и ESI-MS-анализом. Спектры излучения 
соединений в твердом состоянии регистрировали с помощью детектора счета фотонов. Экспериментальные параметры интенсивности $\Omega_{\lambda} \quad 4 f-4 f$-переходов определяли с использованием хорошо известных уравнений для излучаемой мощности, $\Omega_{\lambda}^{\mathrm{P}}$, и счета фотонов, $\Omega_{\lambda}^{\mathrm{C}}$. Различия между $\Omega_{\lambda}^{\mathrm{P}}$ и $\Omega_{\lambda}^{\mathrm{C}}$ являются систематическими, причем $\Omega_{\lambda}^{\mathrm{P}}>\Omega_{\lambda}^{\mathrm{C}}$ и $\Omega_{2}^{\mathrm{P}}$ на $3.4-3.9 \%$ больше, чем $\Omega_{2}^{\mathrm{C}}$, в то время как $\Omega_{4}^{\mathrm{P}}$ на $15.0-15.6 \%$ больше, чем $\Omega_{4}^{\mathrm{C}}$. Эти небольшие и систематические различия обусловлены малой ковалентностью и слабым влиянием поля лигандов, что обеспечивает отличную линейную корреляцию между параметрами $\Omega_{\lambda}^{\mathrm{P}}$ и $\Omega_{\lambda}^{\mathrm{C}}$. Итак, для этих соединений были получены поправочные коэффициенты, позволяющие проводить преобразования $\Omega_{2}^{\mathrm{P}} \leftrightarrow \Omega_{2}^{\mathrm{C}}$ и $\Omega_{4}^{\mathrm{P}} \leftrightarrow \Omega_{4}^{\mathrm{C}}$. Кроме того, из-за эффектов экранирования, $4 f-4 f$-переходы дают очень узкие линии излучения, поэтому площади, рассчитанные по спектру, зарегистрированному в длинах волн, практически совпадают с площадями, полученными из спектра, преобразованного в волновые числа по Якоби. Поэтому мы предлагаем при вычислении параметров интенсивности обращать и особое внимание на тип регистрации и даем поправочные коэффициенты для параметров, определяемых по спектрам, записанным со счетом фотонов, но рассчитанным в предположении регистрации излучаемой мощности. Такие поправочные коэффициенты могут быть использованы практически для любого комплекса $\mathrm{Eu}^{3+} \mathrm{c}$ координированными атомами кислорода или азота.

\section{Вклад авторов}

L. Blois: концептуализация, методология, исследование, формальный анализ, визуализация, курация, написание - оригинальный проект, Написание - обзор и редактирование. A.N. Carneiro Neto: концептуализация, методология, формальный анализ, валидация, написание оригинальный проект, Написание - обзор и редактирование. R.L. Longo: формальный анализ, валидация, написание - оригинальный проект, написание - обзор и редактирование. I.F. Costa: методология, визуализация, курация, написание - оригинальный проект, написание - обзор и редактирование. T.B. Paolini: методология, написание - обзор и редактирование. H.F. Brito: концептуализация, методология, визуализация, курация, надзор, привлечение финансирования, администрирование проекта, написание оригинального проекта, написание обзор и редактирование. O.L. Malta: концептуализация, методология, формальный анализ, визуализация, надзор, написание - оригинальный проект, написание - обзор и редактирование.

\section{Благодарности}

L. Blois благодарит Prof. Erick Bastos и Frank Quina (Instituto de Química - USP) за предоставление Edinburgh FLS980 Fluorometer. Авторы благо- дарны Analytical Central of the Institute of Chemistry (Central Analítica - IQUSP) за элементный и массспектрометрический анализ. Эта статья посвящена профессору Марине Поповой.

\section{Финансирование работы}

Авторы выражают благодарность бразильским финансирующим учреждениям: Conselho Nacional do Desenvolvimento Científico e Tecnológico (CNPq), Coordenação de Aperfeiçoamento de Pessoal de Nível Superior (CAPES) и Fundação de Amparo à Pesquisa do Estado de São Paulo (FAPESP). L. Blois благодарен FAPESP за стипендию Ph.D. (Grant 2020/16795-6). A.N. Carneiro Neto благодарен SusPhotoSolutions - Soluções Fotovoltaicas Sustentáveis, CENTRO-01-0145-FEDER-000005. R.L. Longo благодарен за частичную финансовую поддержку в рамках грантов: Pronex APQ-0675-1.06/14, INCTNANOMARCS APQ-0549-1.06/17, APQ-1007-1.06/15 и стипендию CNPq-PQ (Grant 309177/2018-9). I.F. Costa благодарен $\mathrm{CNPq}$ за стипендию для докторантуры (Grant 151623/2020-1). H.F. Brito благодарен CNPq за исследовательский грант (306951/2018-5).

\section{Конфликт интересов}

Авторы заявляют, что у них нет известных конкурирующих финансовых интересов или личных отношений, которые могли бы повлиять на работу, описанную в этой статье.

\section{Список литературы}

[1] A.G. Bispo-Jr, L.F. Saraiva, S.A.M. Lima, A.M. Pires, M.R. Davolos. J. Lumin., 237, 118167 (2021).

[2] J.-C.G.C.G. Bünzli, C. Piguet. Chem. Soc. Rev., 34, 1048 (2005).

[3] P. Caravan, J.J. Ellison, T.J. McMurry, R.B. Lauffer. Chem. Rev., 99, 2293 (1999).

[4] M.N. Popova, E.P. Chukalina, B.Z. Malkin, S.K. Saikin. Phys. Rev. B, 61, 7421 (2000).

[5] N. Agladze, M. Popova, G. Zhizhin, V. Egorov, M. Petrova. Phys. Rev. Lett., 66, 477 (1991).

[6] B.Z. Malkin, A.R. Zakirov, M.N. Popova, S.A. Klimin, E.P. Chukalina, E. Antic-Fidancev, P. Goldner, P. Aschehoug, G. Dhalenne. Phys. Rev. B, 70, 075112 (2004).

[7] N.I. Agladze, M.N. Popova. Solid State Commun., 55, 1097 (1985).

[8] M.N. Popova, S.A. Klimin, E.P. Chukalina, E.A. Romanov, B.Z. Malkin, E. Antic- Fidancev, B.V. Mill, G. Dhalenne. Phys. Rev. B, 71, 024414 (2005).

[9] G.F. de Sá, O.L. Malta, C. de Mello Donegá, A.M. Simas, R. L. Longo, P.A. Santa-Cruz, E.F. da Silva. Coord. Chem. Rev., 196, 165 (2000).

[10] H.F. Brito, O.M.L. Malta, M.C.F.C. Felinto, E.E. de S. Teotonio. In Chem. Met. Enolates, ed. by J. Zabicky, 1st ed. (Wiley, Chichester, 2009), p. 131-184. 
[11] A.N. Carneiro Neto, E.E.S. Teotonio, G.F. de Sá, H.F. Brito, J. Legendziewicz, L.D. Carlos, M.C.F.C. Felinto, P. Gawryszewska, R.T. Moura Jr., R.L. Longo, W.M. Faustino, O.L. Malta. In: Handb. Phys. Chem. Rare Earths, Vol. 56, ed. by J.-C.G. Bünzli, V.K. Pecharsky (Elsevier, 2019), p. $55-162$.

[12] I.P. Assunção, A.N. Carneiro Neto, R.T. Moura, C.C.S. Pedroso, I.G.N. Silva, M.C.F.C. Felinto, E.E.S. Teotonio, O.L. Malta, H.F. Brito. ChemPhysChem, 20, 1931 (2019).

[13] O.L. Malta. Chem. Phys. Lett., 87, 27 (1982).

[14] R.T. Moura Jr., A.N. Carneiro Neto, R.L. Longo, O.L. Malta. J. Lumin., 170, 420 (2016).

[15] P.R.S. Santos, D.K.S. Pereira, I.F. Costa, I.F. Silva, H.F. Brito, W.M. Faustino, A.N. Carneiro Neto, R.T. Moura, M.H. Araujo, R. Diniz, O.L. Malta, E.E.S. Teotonio. J. Lumin., 226, 117455 (2020).

[16] D.O.A. Dos Santos, L. Giordano, M.A.S.G. Barbará, M.C. Portes, C.C.S. Pedroso, V.C. Teixeira, M. Lastusaari, L.C.V. Rodrigues. Dalt. Trans., 49, 16386 (2020).

[17] D.L. Fritzen, L. Giordano, L.C.V. Rodrigues, J.H.S.K. Monteiro. Nanomaterials, 10, 1 (2020).

[18] M. Suta, C. Wickleder. J. Lumin., 210, 210 (2019).

[19] K. Binnemans. Coord. Chem. Rev., 295, 1 (2015).

[20] J. Kai, M.C.F.C. Felinto, L.A.O. Nunes, O.L. Malta, H.F. Brito. J. Mater. Chem., 21, 3796 (2011).

[21] L.B. Guimarães, A.M.P. Botas, M.C.F.C. Felinto, R.A.S. Ferreira, L.D. Carlos, O.L. Malta, H.F. Brito. Mater. Adv., 1, 1988 (2020).

[22] A.S. Souza, L.A.O. Nunes, I.G.N. Silva, F.A.M. Oliveira, L.L. Da Luz, H.F. Brito, M.C.F.C. Felinto, R.A.S. Ferreira, S.A. Júnior, L.D. Carlos, O.L. Malta. Nanoscale, 8, 5327 (2016).

[23] S.I. Weissman. J. Chem. Phys., 10, 214 (1942).

[24] G.S. Ofelt. J. Chem. Phys., 37, 511 (1962).

[25] B.R. Judd. Phys. Rev., 127, 750 (1962).

[26] C.K. Jørgensen, B.R. Judd. Mol. Phys., 8, 281 (1964).

[27] S.F. Mason, R.D. Peacock, B. Stewart. Mol. Phys., 30, 1829 (1975).

[28] B.R. Judd. J. Chem. Phys., 70, 4830 (1979).

[29] O.L. Malta, H.J. Batista, L.D. Carlos. Chem. Phys., 282, 21 (2002).

[30] O.L. Malta, H.F. Brito, J.F.S. Menezes, F.R.G. e Silva, S. Alves, F.S. Farias, A.V.M. de Andrade. J. Lumin., 75, 255 (1997).

[31] O.L. Malta, H.F. Brito, J.F.S. Menezes, F.R.G.E. Silva, C.D. Donega, S. Alves. Chem. Phys. Lett., 282, 233 (1998).

[32] G.B.V. Lima, J.C. Bueno, A.F. da Silva, A.N. Carneiro Neto, R.T. Moura, E.E.S. Teotonio, O.L. Malta, W.M. Faustino. J. Lumin., 219, 116884 (2020).

[33] H. Song, G. Liu, C. Fan, S. Pu. J. Rare Earths, 39, 460 (2021).

[34] D. Zhang, Y. Zhang, Z. Wang, Y. Zheng, X. Zheng, L. Gao, C. Wang, C. Yang, H. Tang, Y. Li. J. Lumin., 229, 117706 (2021).

[35] R. Adati, J. Monteiro, L. Cardoso, D. de Oliveira, M. Jafelicci, M. Davolos. J. Braz. Chem. Soc., 30, 1707 (2019).

[36] E.E.S. Teotonio, H.F. Brito, M.C.F.C. Felinto, C.A. Kodaira, O.L. Malta. J. Coord. Chem., 56, 913 (2003).

[37] C. Yang, J. Xu, J. Ma, D. Zhu, Y. Zhang, L. Liang, M. Lu. Photochem. Photobiol. Sci., 12, 330 (2013).

[38] Z. Li, Z. Hou, D. Ha, H. Li. Chem. -- An Asian J., 10, 2720 (2015).

[39] X.H. Zhao, K.L. Huang, F.P. Jiao, S.Q. Liu, Z.G. Liu, S.Q. Hu. J. Phys. Chem. Solids, 68, 1674 (2007).
[40] R.D. Adati, M.R. Davolos, M. Jafelicci, S.A.M. Lima, C. Viegas. Phys. Status Solidi Curr. Top. Solid State Phys., 6, 7 (2009).

[41] E.E.S. Teotonio, G.M. Fett, H.F. Brito, W.M. Faustino, G.F. de Sá, M.C.F.C. Felinto, R.H.A. Santos. J. Lumin., 128, 190 (2008).

[42] H.F. Brito, O.L. Malta, J.F.S. Menezes. J. Alloys Compd., 303-304, 336 (2000).

[43] J. Mooney, P. Kambhampati. J. Phys. Chem. Lett., 4, 3316 (2013).

[44] J. Mooney, P. Kambhampati. J. Phys. Chem. Lett., 5, 3497 (2014).

[45] L.W. McDonald, J.A. Campbell, S.B. Clark. Anal. Chem., 86, 1023 (2014).

[46] W.T. Carnall, H. Crosswhite, H.M. Crosswhite, Energy Level Structure and Transition Probabilities in the Spectra of the Trivalent Lanthanides in LaF (Argonne, IL, United States, 1978).

[47] R. Van Deun, K. Binnemans, C. Görller-Walrand, J.L. Adam. J. Phys.: Condens. Matter, 10, 7231 (1998).

[48] G. Blasse, B.C. Grabmaier, Luminescent Materials (Springer Berlin Heidelberg, Berlin, Heidelberg, 1994).

Перевод с английского К. Журавлев 